\title{
LA PROGRAMACIÓN NEUROLINGÜÍSTICA EN LOS DIRECTIVOS DE LAS ORGANIZACIONES EDUCATIVAS PERUANAS EN TIEMPOS DE PANDEMIA
}

\author{
Jorge Luis Romero Chacín* \\ https://orcid.org/0000-0002-5738-7504 \\ Rosario Mireya Romero Parra** \\ https://orcid.org/0000-0002-1319-1171 \\ Luis Andrés Barboza Arenas*** \\ https://orcid.org/0000-0001-7793-1258
}

\section{RECIBIDO: Febrero 2021 / ACEPTADO: Mayo 2021 / PUBLICADO: Septiembre 2021}

\begin{abstract}
Como citar: Romero Chacín, Jorge; Romero Parra, Rosario; Barboza Arenas, Luis. (2021). La Programación Neurolingüística en los directivos de las organizaciones educativas peruanas en tiempos de pandemia. Telos: revista de Estudios Interdisciplinarios en Ciencias Sociales, 23 (3), Venezuela. (Pp. 514-531).

DOI: www.doi.org/10.36390/telos233.02
\end{abstract}

\section{RESUMEN}

La PNL se proyecta como una herramienta de perfeccionamiento colectivo e individual en el marco de la excelencia, que favorece la humanización y sensibilidad de este mundo neurotizado por las diferentes barreras comunicacionales y paradigmas culturales y sociales; con el objetivo de optimizar nuestras esferas de actuación. La finalidad del presente artículo fue analizar el conocimiento acerca de la Programación Neurolingüística que posee el personal directivo de las escuelas de Educación Básica del distrito los olivos de la provincia de lima, Perú en los actuales tiempos de pandemia. El estudio se fundamentó con los planteamientos teóricos de Douat (2017), Deminco (2019), Muñoz (2019), Redford (2017), Romero et al. (2015), entre otros. Se utilizó una metodología descriptiva con diseño no experimental. La muestra estuvo constituida por 58 sujetos, a los cuales se les aplicó un cuestionario. Los resultados muestran que los directivos poseen un mediano conocimiento de la PNL y con respecto a la comunicación, la media aritmética es de 0,188 categorizándose en muy baja, según el baremo establecido, lo cual indica que el personal directivo de los colegios nacionales de educación primaria del distrito Los Olivos de la provincia de Lima, poseen muy bajo conocimiento acerca de la comunicación, resultando un aspecto negativo en cuanto al desenvolvimiento gerencial del personal referido, ya que no conocen en su totalidad esta característica fundamental para el desarrollo de la PNL

\footnotetext{
Doctor en Ciencias de la Educación. Profesor de la Universidad Continental, Cusco, Perú. Correo electrónico: jromeroch@continental.edu.pe

** Doctora en Ciencias de la Educación. Profesora de la Universidad Continental, Lima, Perú. Investigadora con código Renacyt: P0162004 del CONCYTEC. Correo electrónico: rosarioromeroparra@gmail.com

*** Doctor en Ciencias de la Educación. Universidad Tecnológica del Perú. Correo electrónico: Luissbarbozza@gmail.com
} 
en todos los ámbitos. Por ello se propone, el uso de la PNL, para que posibilite el desarrollo de habilidades con las que se puedan concebir resultados que optimicen la comunicación del personal directivo de los colegios referidos en los actuales tiempos de pandemia.

Palabras clave: comunicación; estrategias; neurolingüística; pandemia; programación.

\section{Neuro-linguistic programming in the directors of Peruvian educational organizations in times of pandemic}

\section{ABSTRACT}

NLP is projected as a tool for collective and individual improvement within the framework of excellence, which favors the humanization and sensitivity of this world neurotized by the different communicational barriers and cultural and social paradigms, aiming to optimize our spheres of action. The purpose of this article was to analyze the knowledge about Neurolinguistic Programming possessed by the management staff of Basic Education schools in the district of Los Olivos in the province of Lima, Peru, in the current times of pandemic. The study was based on the theoretical approaches of Douat (2017), Deminco (2019), Muñoz (2019), Redford (2017), Romero et al. (2015), among others. A descriptive methodology with a non-experimental design was used. The sample consisted of 58 subjects to whom a questionnaire was applied. The results show that managers have a medium knowledge of NLP and with respect to communication, the arithmetic mean is 0.188 , categorized as very low, according to the established scale, which indicates that the management staff of the national primary schools of the Los Olivos district of the province of Lima, have very low knowledge about communication, resulting in a negative aspect regarding the managerial development of the referred staff since they do not know in full this fundamental characteristic for the development of NLP in all areas. Therefore, NLP is proposed to enable the development of skills with which results can be conceived to optimize the communication of the management staff of the referred schools in the current times of pandemics.

Keywords: communication; strategies; neurolinguistics; pandemic; programming.

\section{Introducción}

La Programación Neurolingüística (PNL), es un sistema estratégico o modelo que, fundamentado en la comunicación, consiente el saber de cómo construimos nuestros sentimientos y pensamientos, y de esta manera precisa, podemos aprovechar habilidades ajenas y propias para concebir permutas efectivas que facilitan por consecuencia cualquier conducta humana (Romero et al., 2015).

En efecto, la PNL ha crecido rápidamente y en la actualidad está generando interés en áreas cómo la dirección y la preparación, analizando los principios y técnicas de esta, para originar cambios fundamentales y duraderos en el comportamiento, tanto en el trabajo como en la vida personal.

Además, según Maldonado-Vicente (2017), la PNL persigue que el ser humano encause su realización personal y profesional y considere su libertad, por lo cual le aproxima a percibir la manera en la cual cada individuo organiza sus vivencias, en relación con su propia identidad, 
La Programación Neurolingüística en los directivos de las organizaciones educativas peruanas en tiempos de pandemia.

así como para con los demás y el ambiente que le rodea, para así hacer propicio de manera particular, la evolución que le admitirá alcanzar el éxito, enalteciendo su autoestima, optimizando la apariencia de sí mismo mediante la autoaceptación, produciendo permutas para aumentar el potencial creativo, obtener una eficaz intervención de las emociones, gestionar de manera cabal las capacidades mentales, los recursos intelectuales, las destrezas y habilidades para poder desarrollar muchas otras competencias.

Por otra parte, la PNL muestra cómo se puede pensar mejor, a través del desarrollo del cerebro triuno, así como lograr efectos superiores, instruye como comunicarse de forma intrínseca y extrínsecamente, de manera que logre establecerse la discrepancia entre la insuficiencia y la excelencia (Deminco, 2019). Ayudando así a generar nuevos comportamientos y a modificar conductas no deseadas, facilitando el logro de objetivos.

Así mismo, este estudio brindará la manera y los medios a los directivos de alcanzar la perfección organizacional de las instituciones educativas puesto que, según Dilts y DeLozier (2016), por medio de la Programación Neurolingüística, se aprende a comunicar con los tres modelos de sistemas perceptivos (auditivas, visuales y cenestésicas), para conseguir sintonía con ellos, proporcionar la comunicación, la plática, relación y aproximación propicia.

Al mismo tiempo, Rojas y Calle (2019), plantean que la Programación Neurolingüística permite favorecer la permuta para alcanzar objetivos relevantes de manera más sencilla, debido a que se basa en el análisis experiencial de los métodos del pensamiento, logrando colaborar en el proceder de los individuos en el área gerencia educativa, debido a que esta logra desarrollar a totalidad el funcionamiento del cerebro logrando controlar la inteligencia emocional de los gerentes en las organizaciones educativas, beneficiando a todos sus actores y por consiguiente a todos en general.

En el Perú el COVID-19 llegó el 11 de marzo de 2020, fecha en la cual el presidente, del momento, Martin Vizcarra comunica de los casos iniciales en el Perú. En consecuencia, el estado se encontraba en situación de emergencia, en los últimos meses la población y comunidades, estaban golpeados por esta enfermedad de COVID-19, llevando al colapso al sistema sanitario. En ese orden de ideas, Délano (2020), expresa como en los últimos meses se ha visto una nueva enfermedad viral generando colapso en los sistemas sanitarios y creando caos y miedos en las personas, aquejando en primer lugar a Wuhan, China, y que actualmente complica a todo el mundo. Sin embargo, las actividades no se detienen, incluyendo las académicas, por ello es necesario precisar y solventar las dificultades que puedan estar presentando tanto los directivos como el estudiantado, en el ámbito educativo en estos tiempos de pandemia.

En consecuencia, considerando la transmisión del COVID-19 y las medidas de cuidado efectuadas a manera general, el empleo de modelos de instrucción no convenidos en los regímenes de enseñanza primaria se ha transformado en una táctica forzosa para el acatamiento de propósitos y compromisos (Arbeláez-Campillo y Villasmil, 2020; López, 2020; RosarioRodríguez et al., 2020).

Empero, atendiendo el acoplamiento del COVID-19, a las instancias académicas, científicas y psíquicas (originada por la ejecución infranqueable de modelos de instrucción en tiempos remotos y problemas en el progreso de políticas de ejecución y formación eficaz) han contribuido a que el estudiantado perciba problemas y obstáculos, aparte de asumir una forma efectiva por 
el empleo de las TIC en la instrucción (Sanz et al., 2020). En consecuencia, organizaciones mundiales como la UNESCO (2020), persuaden a efectuar una aproximación a partir lo conceptual y supuestos teóricos con el fin de demostrar las operaciones, instrucciones y recorridos que convienen asumir en favor de la colectividad académica, haciendo énfasis en el alumnado. Todo ello, con el fin de verificar elementos que inciden en una buena experiencia de instrucción a distancia y admitan perfeccionar los efectos prometidos (Monasterio y Briceño, 2020); en circunstancias donde los procedimientos adoptados están en manos de las competencias, recursos y particularidades de formación con las que presenta cada nación y sus regímenes de formación primaria.

En esa línea de investigación, los descubrimientos más importantes muestran que los alumnos asumieron dificultades afines a elementos estresantes, como el exceso de actividades escolares, pésima ejecución de cursos de forma virtual, menoscabo de alineación de sus instituciones, escasas empatía de sus catedráticos, entre otros aspectos. Los resultados encontrados logran aumentos en indicios de depresión, ansia, estrés postraumático, entre otros (Romero, 2020; Velavan y Meyer, 2020; Blanco et al., 2021; Montiel et al., 2021). Es por ello que, las operaciones que logren efectuar las instituciones en favor del bienestar emocional de sus alumnos en los actuales momentos y pos-pandemia pudieran suavizar las secuelas psicológicas del COVID-19 y beneficiar el avance de la sociedad.

En ese sentido, las posibles consecuencias adversas de la extensa cesación de las clases presenciales en la formación de los alumnos y los peligros procedentes de ello, como el engrandecimiento de las fisuras de la enseñanza y de la deserción estudiantil (Cobo-Rendón et al., 2020), pueden ser suavizadas con la aplicación de estrategias de PNL.

En este orden, este estudio tiene como finalidad analizar el conocimiento acerca de la Programación Neurolingüística que posee el personal directivo de las escuelas de educación primaria del distrito Los Olivos de la provincia de Lima, Perú, para enfrentar las dificultades en los actuales tiempos de pandemia.

\section{Referentes conceptuales}

\section{Bases de la Programación Neurolingüística}

Las bases o hipótesis son los fundamentos primordiales de la PNL, éstos construyen todo el sustentáculo de la vinculación de elementos 0 soportes que establece como se distingue el comportamiento, la manera de encarar la información y la voluntad propia de cada ser humano. Dichas bases según Carrión (2010), conforman el modelo aprobatorio para trabajar la PNL como habilidad y como erudición aplicada, su perspicacia y su interacción con el medio en el que nos desenvolvemos.

\section{Bases Neurológicas}

La PNL instruye a retomar los componentes esenciales de cada esquema, añadiéndole cualidades distintas de acuerdo a cada persona; puesto que cada cerebro posee una manera propia de procesar. Al mismo tiempo, afianza de la ciencia de los ordenadores, la naturalidad de la información adecuada para establecer y pronto explorar la dependencia existente en su distribución interna y así poder ordenarla y cambiarla en comportamiento (Deminco, 2019). Por lo tanto, tener éxito es una señal de que es aprendido y radica en brindar la posibilidad de 
La Programación Neurolingüística en los directivos de las organizaciones educativas peruanas en tiempos de pandemia.

equiparse con los mejores "software" y educarse a utilizarlos con el mayor efecto, y así convertirse en conductores felices en materia de nuestras redes neurales.

El Cerebro Triuno: El cerebro humano se encuentra constituido por tres sistemas neurales conectados entre sí y cada uno de ellos tiene su determinada y exclusiva inteligencia, así como su propio desempeño. Estas tres mentes o formaciones expuestas por MCLEAN comprenden: el Complejo Reptílico o Sistema - R, el Sistema Límbico y la Corteza o Neocortex (hemisferios cerebrales izquierdo y derecho). A cada una de estas estructuras corresponde un proceso de transformaciones significativas que han conducido nuestra evolución como especie (Douat, 2017).

Complejo reptílico o sistema - R. En la base del sistema nervioso adosado justo al lado de la silla turca del cráneo, tenemos un cerebro primitivo el cual maneja aquellos ejercicios inconscientes como lo es el apetito, el sueño, la sed, la respiración, el sexo y el resto del comportamiento instintivo y acostumbrado; es decir, su acción es espontánea y automática, cumpliendo sus funciones sin intervención del pensamiento. Mientras que, el cerebro límbico, es el segundo cerebro, se denomina también cerebro mamífero o emocional. Se encuentra ubicado en la parte posterior del rostro, envuelve al cerebro más primitivo (reptil) y se halla acoplado a la neocorteza (Flores, 2018). Este cerebro rige todas las emociones, las respuestas metabólicas y los sentimientos: el amor, la rabia, la alegría, el miedo, la tristeza, etc. Por tal razón, éste es más consciente que el anterior y es el que determina las preferencias. Por último, está la neocorteza, la cual es el tercer y último cerebro, el cerebro nuevo, esa masa gris que evolucionó con gran (Gamboa et al., 2017). El mismo, está fraccionado en dos mitades (hemisferio rapidez cerebral izquierdo y derecho). Cada mitad desempeña distintos ejercicios según la práctica del intelecto, pero el trabajo principal de esa dualidad es deliberar, uno lo realiza con representaciones relacionadas a los sentidos y el otro con palabras. Cabe resaltar, que el hemisferio cerebral izquierdo maneja la parte derecha del organismo y se ha demostrado que es predominante hablador, lógico, matemático, analizador, mientras el derecho es creativo, intuitivo, artístico, sintetizador.

Bilateralidad Cerebral: Los hemisferios cerebrales muestran dos modalidades de pensamiento, representadas de forma verbal (hemisferio izquierdo) y la otra forma no verbal (hemisferio derecho). Normalmente, la idea de que existen dos modos periféricos de pensamiento apunta que la instrucción, repose mediante la entrevista o la reproducción, perturbando primeramente a una de las dos mitades cerebrales (Redford, 2017). Por tal motivo, el aprendizaje en cualquier contenido académico será más positivo en la mesura que sean estimulados ambos modos mediante la manifestación variada de los contenidos y a través del uso de un currículo el cual provoque el progreso de los dos hemisferios de forma equilibrada.

\section{Bases Lingüísticas}

Cuando se habla, se realiza una sucesión de alternativas, sobre el modo en que se expresa la práctica en un periodo categórico, generalmente, estas alternativas son tomadas de forma involuntaria y obedece a todos las delimitaciones, experiencias, asedios, etcétera, lo cual significa que acata unas normas. De este modo, la PNL instruye a hacer sensatas esas normas a transformar los condicionamientos y a ocuparse para vencer los asedios mentales, modificando un patrón dañado por uno beneficiado y colmado de dominio. De esta forma, los 
individuos requieren la comprensión de un idioma para comunicarse y conjuntamente emplean un conjunto de signos que aprueban la reciprocidad de información.

La Comunicación: Se divide en dos elementos: manual y analógico. En el elemento digital la comunicación, es la distinción de las palabras, los símbolos, es decir, lo que expresamos. El elemento analógico, representa a la particularidad y manera de cómo lo expresamos (Sánchez, 2020). Aunado a esto, se puede decir que, en la comunicación, de acuerdo al segundo aspecto mencionado con anterioridad, intervienen fundamentalmente la voz (intensidad, tono, cadencia, entre otros) y la fisiología (respiración, postura, entre otros).

En este orden de ideas, el lenguaje manual cuenta con una coordinación lógica fuertemente complicada, vigorosa, que priva un significado adecuado en el área de la correspondencia, mientras que el lenguaje analógico tiene el significado, pero no la coordinación apropiada para el esclarecimiento indiscutible en el ambiente de las diplomacias (Vanga y Fernández, 2016). Cabe resaltar que, manual es aquello que se representa mediante dígitos universalmente establecidos, estructuran para nombrar o identificar algo, aunque no exista similitud alguna entre la expresión y el objeto. Y el analógico, hace referencia a la forma de comunicación no verbal, es decir, todo aquello que se haga virtualmente, lo cual es bastante utilizado actualmente por motivo del aislamiento social provocado por la pandemia. En relación con lo anterior, la parvedad de armonizar ambas lenguas, el individuo, sea como destinatario o como emisor, deberá trasladar continuamente de uno a otro, y, al hacer esto, enfrentarse con varios y curiosos dilemas.

Por otra parte, los principales compromisos de la PNL se fundamentaron en la comunicación y la lengua, procurada la relevancia de que, en todo asunto humano, se posee el aspecto comunicacional. Por lo cual, para alcanzar una excelente comunicación es imperioso: ser flexible, ser respetuoso al momento de comunicarnos con otro individuo, reconocer que no siempre tenemos la verdad en las manos, no hay que calificar, contrastar ni desfavorecer a los demás, se deben examinar otras vías de comunicación, así como conllevar las vivencias, ser conscientes de que no siempre se conoce todo, saber admitir las equivocaciones y aprender de ellas para continuar adelante.

Relaciones Lógico-semánticas: Las relaciones Lógico-semánticas son las reflexiones que los hablantes crean sobre el vínculo lógico empleado en su lenguaje: Complejidad: las relaciones entre sí son constituidas por las representaciones expresadas (Redford, 2017). Lo cual quiere decir, que esos métodos comprometen a que los hablantes puedan efectuar innovaciones partiendo de un modelo sintáctico explícito y constantemente según su práctica particular. Lo dicho anteriormente, accede entonces un modo específico de constituir el lenguaje, con una disposición habitual asentada en la lengua, y exclusivamente fundada en el habla.

\section{Propuestas fundamentales de la PNL}

El nivel de competitividad en un individuo cuyo fin es generar innovaciones rápidas y positivas en otras personas dependerá del modelo del mundo que se tenga, por es importante analizar tres elementos básicos que presenta la PNL como propuestas, como lo son los canales de comunicación, sistemas de representación y el metaconocimiento.

Canales de comunicación: En la comunicación de los seres humanos no solo es lenguaje y hábito, sino que hay una naturaleza interior que la reconoce como un elemento, dicha naturaleza 
La Programación Neurolingüística en los directivos de las organizaciones educativas peruanas en tiempos de pandemia.

son los distintos conductos o canales usados para exteriorizar nuestras opiniones sobre el universo.

En este contexto, Deminco (2019), establece el patrón que cada individuo posee del universo es un símbolo mental que obedece a sus vivencias, sabiduría, temperamento, etcétera. Por tal razón, al proporcionar nuestra opinión lo hacemos por medio de numerosos canales; entre los cuales se pueden mencionar a continuación: Verbal: consiste en la forma de hablar, los predicados que utilizamos, los proverbios, así como el modo de ordenar el lenguaje. Gestual: se refiere a la actitud corporal, la compostura, señas, muecas, es decir, todo aquello que se muestre con el cuerpo. Modo de dominar el entorno: representa las proximidades y distancias que creamos con los individuos. Colores: simboliza la vibración de cada color; dicho de otro modo, exterioriza el sentir del individuo. Sonidos: la melodía y tonos con los que expresamos las palabras a veces denotan más que las palabras mismas.

Sistemas de Representación: Para Álvarez et al. (2019), aun cuando teóricamente hay tantos sistemas representacionales como sentidos se tiene, se pude concluir que se trabaja con tres sistemas: de vista, de audición, kinestésico (olfato, gusto y tacto). El sistema representativo de la vista se manifiesta cuando se piensa en fotografías mentales, es decir cuando se conmemoran retratos imprecisos y específicos como números y letras. Mientras que, el sistema de audición es el que permite recordar músicas o conversaciones, es decir, es el que aprueba oír en los pensamientos voces, ecos, melodías. $Y$ el kinestésico se refiere a procesar la información asociándola a las emociones y movimientos del cuerpo.

Metaconocimiento: La discrepancia entre un individuo que tiene el metaconocimiento de una destreza y de otro que no la posee, es que el primero puede volver a crear sobre lo aprendido porque ha aprovechado intrínsecamente, el como del qué. Mientras el segundo, redundará infaliblemente por ensayo y error el qué, sin saber el cómo, descubriendo una progresiva restricción debido a que no podrá optimizar la construcción, tampoco será eficaz al educarlo, y si se le muestra un conflicto no sabrá resolverlo debidamente (Douat, 2017). El metaconocimiento se refiere al potencial que poseen los seres humanos para manifestar cómo se han preparado para la vida, es decir, cómo han alcanzado los conocimientos, habilidades o destrezas, y así poder revelarle a alguien el modo de cómo obtuvo tal habilidad y la manera más poderosa de emplearla para colocarla en práctica.

\section{Estrategias de PNL}

Como referentes conceptuales del presente artículo, se tienen las estrategias de PNL que se pueden implementar en las organizaciones educativas en tiempos de pandemia. Según, Morles et al. (2017), una estrategia es un proceso mental que lleva una respuesta, manifestándose con una sucesión de particularidades encadenadas. En PNL una estrategia coloca en movimiento el sistema neurológico (Carrión, 2010). Considerando que todas las personas tienen conjuntos de estrategias específicos, para efectuar varias cosas; existen estrategias de comunicación, relajación, estrategias para elegir la comida, para motivar, entre otras. Considerando, que en ciertas ocasiones dichas estrategias tengan como resultado ser enormemente operativas y otras verdaderamente ineficientes. Por ello a continuación se presentan las estrategias más pertinentes para afrontar estos tiempos de pandemia y que deben 
tener presente las organizaciones educativas, las cuales son expuestas por Romero et al. (2015).

Relajación: El aprender y aplicar estrategias de relajación, ofrece a los individuos, una gran oportunidad para conservar su cuerpo concertado y reducir el peligro de perturbaciones ascendentes, proporcionándole también el aprendizaje, la asimilación de este y las respuestas oportunas para enfrentar el contexto que, en un determinado momento, puede surgir como perverso, como es el caso de pandemias (Monterrosa et al., 2020).

Modelaje: Las estrategias de este tipo se crean de manera natural como segmento de la tendencia modelante. Sólo tienes que observar a un niño creciendo en una casa donde el padre es un entusiasta del fútbol, para ver la tendencia natural del niño en el modelado del comportamiento. Un efecto similar puede verse cuando el niño está rodeado de artistas, cocineros, nadadores, ciclistas, o lo que sea. También la televisión tiene influencia, y el comportamiento negativo antisocial tiene tantas probabilidades de ser modelado como el comportamiento positivo, como lo plantea Gamboa et al. (2017).

Sincronización: Es el procedimiento a través del cual se origina un profundo vínculo entre los niveles conscientes e inconscientes de un interlocutor. La sincronización es un reflejo, es revelar un espejo, sin imitación, enviando señales no verbales, que la otra persona pueda igualar esclarecida e inconscientemente como suyas, demostrando para ella un afianzamiento de que ha sido comprendida, aceptada y reconocida (Estupiñan y de Mora, 2017).

Por su parte, al momento de sincronizar, se origina un ambiente de confianza y compañerismo lo cual permite que el interlocutor siga hablando y pueda comunicarse con mayor eficacia. Cuando se sincroniza, se afina la capacidad de escuchar, consiguiendo ser más precisos al dar una opinión; permitiendo finalmente capitanear la relación, guiando la conversación para que así el interlocutor sea en todo momento más espontáneo y abierto.

Anclaje: Consiste en relacionar las conductas que se quieren adquirir a través de estímulos externos sensoriales (López et al., 2020). De este modo, las anclas se pueden colocar intencionadamente u originar de forma espontánea, produciendo así, distintos estados mentales que emiten involuntariamente procesos cerebrales.

Asimismo, un ancla puede ser una expresión, una seña, un olor, una tonalidad, una delicia que nos transporta mentalmente a un momento explícito porque así se creó en algún momento remoto, al entrenarlo de manera consecuente se transforma en una herramienta potenciadora de estados mentales auténticos y de triunfo. Por ejemplo: acariciarse la nariz en cualquier momento que se desee sentir bien; ambas cosas se unifican, y finalmente, el cerebro realiza toda la labor.

Reencuadre: Busca transformar el significado de los hechos, modificando el marco de referencia en el que una persona los percibió. Por consiguiente, cuando el significado cambia, igualmente lo hace el estado afectivo, los argumentos y comportamientos de las personas. Muñoz (2019) expone que el reencuadre consta de dos tipos: 1.- El contenido: se recibe una afirmación y se le otorga otro sentido, por ejemplo, preguntándose, que otro sentido se le daría a esto (vaso medio lleno o medio vacío). En este contexto, el reencuadre según establecen Álvarez et al. (2019), le confiere al individuo observar los problemas, bloqueos conductuales, las conductas ineficaces o cualquier situación de la vida, desde un enfoque actual 0 desde una perspectiva diferente para alcanzar así óptimas opciones de conducta, manteniendo la ganancia secundaria que las mismas le proporcionaban. 
La Programación Neurolingüística en los directivos de las organizaciones educativas peruanas en tiempos de pandemia.

\section{Metodología}

La investigación fue descriptiva. Se recogió información sobre la variable PNL, desde las dimensiones e indicadores en el perímetro donde se desenvuelven, para analizar la PNL que posee el personal directivo de los colegios nacionales de educación primaria del distrito Los Olivos de la provincia de Lima, Perú en los actuales tiempos de pandemia.

La información necesaria para analizar la variable PNL fue recolectada directamente de fuentes originarias a través del diseño de instrumentos para tal fin. El diseño de la investigación fue no experimental, sus mediciones se ejecutaron sin influir en la variable PNL. Al respecto, Hernández et al. (2018), aseveran que el estudio de tipo no experimental es la que se efectúa sin manipular intencionadamente variables, prestar atención a los fenómenos tal y como se desarrollan en su contexto natural para posteriormente examinarlos.

Por último, el estudio se ejecutó con un diseño no experimental de naturaleza transversal o transeccional que según Hernández et al. (2018, p. 270) "el recojo de datos es en un solo momento". En ese sentido, se realizó la recolección de la información pertinente a la variable Programación Neurolingüística, permitiendo el recojo de la información precisa para analizarla como variable en función de sus dimensiones e indicadores.

La población estuvo conformada por 58 individuos pertenecientes a veintinueve directores y veintinueve subdirectores, que se desempeñan en los colegios nacionales de educación primaria del distrito Los Olivos de la provincia de Lima, Perú. La muestra que conforma el estudio es la misma población (censal), por ser finita. La técnica de recolección de datos es la encuesta y el instrumento empleado fue un cuestionario diseñado con Google Forms de 45 preguntas con 5 opciones de respuesta, usando una escala tipo Likert (Siempre, casi siempre, ocasionalmente, casi nunca y nunca). Es importante señalar que, los ítems o preguntas de este abarcan todas las dimensiones e indicadores estudiados sobre PNL.

Para el procesamiento de los datos se elaboró una matriz o tabla de doble entrada donde se alojaron los datos proporcionados por los sujetos en relación

al procesamiento de variables. Se utilizó la estadística descriptiva, debido a que la información se estableció en tablas de distribución frecuencial y porcentual por indicadores para su análisis. Posterior a esto se confronta los resultados, se presentan en medias aritméticas, a través de la cuales se analizan las dimensiones e indicadores de la variable del estudio, por lo que se establece un baremo con sus respectivas categorías de conocimiento y rangos, para precisar el mismo.

\section{Resultados y discusión}

Los resultados se presentan con las teorías que soporta la investigación, al mismo tiempo que se categorizaron según el baremo ponderado. En la tabla 1, se considera que la media aritmética del indicador cerebro triuno es de 0,167 ubicándolo en la categoría muy bajo conocimiento en atención al baremo establecido. Esto indica que el personal directivo colegios nacionales de educación primaria del distrito Los Olivos de la provincia de Lima, en los actuales tiempos de pandemia, posee muy bajo conocimiento sobre el cerebro triuno, siendo este resultado contradictorio con lo planteado por Douat (2017). 
De acuerdo con su naturaleza, según este autor, señala que el cerebro humano es el foco del aprendizaje y es el miembro más transcendental de nuestro sistema nervioso, ya que es el encargado de relacionar, recibir, originar una gran cantidad de indicaciones y acumular, señales, signos e informaciones fundamentales para que sucedan todas las ocupaciones de todo el cuerpo de las personas. Y al no tener conocimiento sobre el desarrollo potencial del cerebro, nunca se podrá desarrollar a cabalidad el mismo.

Por otra parte, la media aritmética del indicador bilateralidad cerebral es de 0,511 ubicándolo en la categoría mediano en el baremo, lo cual indica que existe un conocimiento mediano sobre las dos particularidades de pensamiento: la verbal y la no verbal representada por los hemisferios cerebrales derecho e izquierdo proporcionalmente. Rojas y Calle (2019), determinan que hay dos singularidades lateralizadas de pensamiento y plantean que la instrucción, bien sea mediante de la imitación o conferencia, afecta principalmente a uno de los dos hemisferios. En consecuencia, el amaestramiento de cualquier contenido independiente del área se desarrollará más significativo debido a la activación de ambas modalidades mediante la presentación diversificada del contenido y mediante el empleo de un currículo que incite el desarrollo de los dos hemisferios de manera balanceada.

Con respecto al indicador comunicación, la media aritmética es de 0,188 categorizándose en muy bajo, lo cual indica que el personal directivo de los colegios nacionales de educación primaria del distrito Los Olivos de la provincia de Lima, en los actuales tiempos de pandemia, poseen muy bajo conocimiento acerca de la comunicación. Frente a este escenario; como se sabe; los primeros estudios de la PNL de Blander y Grinder citados por Romero et al. (2015), tuvieron basadas en el lenguaje y la comunicación, debido a la importancia de que en todo proceso humano tiene el aspecto comunicativo. Ya sea a nivel externo, con los demás o a nivel interno, es decir con nosotros mismos, siempre estamos comunicando, de ahí la vital importancia. La PNL, ha promovido los modelos más sofisticados en técnicas de comunicación, que nos favorecen acercamiento más favorable a los mapas de otras personas, a la identificación de los conflicto internos y externos, así como la mejor manera de resolver todos ellos.

Por otra parte, la media aritmética del indicador relaciones lógico-semánticas es de 0,563 ubicándose en la categoría mediano según baremo. Esto refleja que el personal directivo de los colegios nacionales de educación primaria del distrito Los Olivos de la provincia de Lima, en los actuales tiempos de pandemia, tienen un conocimiento medio de lo que son las relaciones lógico-semánticas, las cuales según Mayorga-Jácome y Rosero-Morales (2016), plantea que los emisores logran ejecutar cambios teniendo como enfoque un modelo sintáctico preciso y eternamente conforme a su ajustada práctica particular, lo cual conviene entonces una forma característica de establecer la expresión, con una estructura frecuente fundamentada en el lenguaje, y específicamente establecida en la lengua. Estos resultados son semejantes a los reportados por Flores (2018) en su investigación, concluyendo que la PNL provee las herramientas necesarias para que los directivos sepan y comprendan mejor las funciones del cerebro, para que puedan influir adecuadamente en el personal docente y estudiantes, logrando entornos educativos agradables y eficientes. 
La Programación Neurolingüística en los directivos de las organizaciones educativas peruanas en tiempos de pandemia.

Tabla 1. Distribución de medias aritméticas de los indicadores y subdimensiones de las Bases de la Programación Neurolingüística.

\begin{tabular}{|c|c|c|c|c|}
\hline Variable & \multicolumn{4}{|c|}{ Programación Neurolingüística } \\
\hline Dimensión & \multicolumn{4}{|c|}{ Bases de la PNL } \\
\hline Subdimensiones & \multicolumn{2}{|c|}{ Neurológicas } & \multicolumn{2}{|c|}{ Lingüísticas } \\
\hline Indicadores & $\begin{array}{c}\text { Cerebro } \\
\text { Triuno }\end{array}$ & $\begin{array}{c}\text { Bilateralidad } \\
\text { Cerebral }\end{array}$ & $\begin{array}{c}\mathrm{La} \\
\text { Comunicación }\end{array}$ & $\begin{array}{l}\text { Relaciones } \\
\text { lógico } \\
\text { semánticas }\end{array}$ \\
\hline $\bar{x}$ Indicador & 0,167 & 0,511 & 0,188 & 0,563 \\
\hline $\bar{x}$ Subdimensión & & 0,339 & 0,3 & \\
\hline $\bar{x}$ Dimensión & \multicolumn{4}{|c|}{0,357} \\
\hline
\end{tabular}

Fuente: Elaboración propia.

En la tabla 2, se puede visualizar que la media aritmética del indicador canales de comunicación es de 0,771 ubicándolo en la categoría alto según el baremo establecido, esto indica que el personal directivo de los colegios nacionales de educación primaria del distrito Los Olivos de la provincia de Lima, posee un alto conocimiento en lo que respecta a los canales de comunicación; esto puede significar un aspecto positivo para la aplicación de la PNL en tiempos de pandemia, ya que según Burgos (2018), la percepción individual de cada persona sobre el mundo es una gráfica mental que depende de su experiencia, cultura, fisiología vivencias, entre otros.

De esta forma, en que la comunicación humana es también costumbre y lengua, y hay un atributo intrínseco que la reconoce como elemento único, esa esencia representa los distintos canales que utilizamos para poner de manifiesto nuestras ideas acerca del mundo que nos rodea.

Así mismo, se observa que la media aritmética del indicador sistemas de representación es de 0,583 , lo cual indica que el personal directivo posee un mediano conocimiento acerca de los sistemas de representación. Esto representa una dificultad para el desarrollo de la comunicación del personal directivo de los colegios nacionales de educación primaria del distrito Los Olivos de la provincia de Lima, ya que según Vanga y Fernández (2016), es muy importante la cohesión entre la expresión y los sistemas perceptivos, debido a que es uno de los recursos que se emplea para enunciar el enfoque de la naturaleza. Para la PNL aún teóricamente hay tantos sistemas representacionales como sentidos tenemos, se puede concluir que se trabaja con tres sistemas: el visual, el auditivo, el kinestésico, olfato, gusto y tacto (Vivas et al., 2019).

Por otra parte, se observa que la media aritmética del indicador metaconocimiento es de 0,281 , ubicándolo en la categoría bajo, esto indica que el personal directivo de los colegios nacionales de educación primaria del distrito Los Olivos de la provincia de Lima, Perú, en los actuales tiempos de pandemia posee un bajo conocimiento acerca del metaconocimiento, siendo este resultado contradictorio con lo planteado por Navarrete (2017), cuando señala que 
el metaconocimiento es la habilidad que tienen las personas para manifestar cómo han aprendido los saberes, es decir, que cuando tenemos una destreza o habilidad, también podremos explicar a alguien la forma de como la obtenemos, la manera más eficiente para su uso y de ejecutarla.

Así mismo, estas derivaciones son parecidas a las enunciadas por Arriagada (2018), quien destaca en su investigación la relevancia de la comunicación y las emociones en el campo educativo, para el logro del bienestar profesional y un buen desenvolvimiento académico, tanto de directivos como docentes de una organización educativa. Estos aspectos son muy importantes en los actuales momentos de pandemia, ya que es vital el desarrollo de una comunicación asertiva y un buen estado emocional para el logro del metaconocimiento en todos los sentidos.

Tabla 2. Distribución de medias aritméticas de los indicadores de la dimensión propuestas fundamentales de la Programación Neurolingüística.

\begin{tabular}{|c|c|c|c|}
\hline Variable & \multicolumn{3}{|c|}{ Programación Neurolingüística } \\
\hline Dimensión & \multicolumn{3}{|c|}{ Propuestas fundamentales de la PNL } \\
\hline Indicadores & $\begin{array}{l}\text { Canales de } \\
\text { comunicación }\end{array}$ & $\begin{array}{l}\text { Sistemas de } \\
\text { representación }\end{array}$ & Metaconocimiento \\
\hline $\bar{x}$ Indicadores & 0,771 & 0,583 & 0,281 \\
\hline $\bar{x}$ Dimensión & \multicolumn{2}{|c|}{0,545} & \\
\hline
\end{tabular}

Fuente: Elaboración propia.

En la tabla 3, se puede observar la media aritmética del indicador anclaje es 0,396, ubicándolo en la categoría bajo según el baremo establecido, esto indica que el personal directivo de los colegios nacionales de educación primaria del distrito Los Olivos de la provincia de Lima en tiempos de la pandemia del coronavirus COVID-19, posee bajo conocimiento acerca de la estrategia anclaje, lo cual se considera un aspecto negativo para el desarrollo de la PNL, ya que Redford (2017), resume los principios y funciones que rigen los anclajes para facilitar una mejor comprensión y aprendizaje.

En este orden de ideas, las anclas son una técnica para asimilar y guardar recuerdos positivos de la vida y suprimir los negativos. La PNL orienta a cómo transformarse en un anclador consciente y positivo, usando los tres canales de comunicación.

Así mismo, se observa que el indicador reencuadre, tiene una media aritmética de 0,375 , lo cual indica según baremo, que el personal directivo posee bajo conocimiento acerca de la estrategia reencuadre, lo cual es negativo para el desarrollo de la PNL, ya que, el reencuadre, según establece Huilca (2017), admite que el individuo observe los problemas, cercos conductuales, las actuaciones ineficaces y cualquiera de las circunstancias de la existencia, a partir de una nueva perspectiva, o comenzando con un enfoque disímil, para lograr excelentes expectativas de actuación. En PNL, se ha desarrollado esta técnica para establecer una correcta comunicación con aquella parte de nosotros mismos que de un modo u otro está creando algún tipo de barreras, bloqueos o trastornos en nuestros propios procesos internos de comunicación. 
La Programación Neurolingüística en los directivos de las organizaciones educativas peruanas en tiempos de pandemia.

Por otra parte, se observa que la media aritmética del indicador submodalidades es de 0,458, ubicándose en mediano, según el baremo establecido, lo cual indica que el personal directivo de los colegios nacionales de educación primaria del distrito Los Olivos de la provincia de Lima en tiempos de la pandemia del coronavirus COVID-19, posee un mediano conocimiento acerca de las submodalidades. Esto indica un aspecto negativo para el desarrollo de la PNL, ya que las submodalidades según como lo plantea Sambrano (2003) citado por Romero et al. (2015), son los códigos operativos del cerebro. Para la PNL son óptimos recursos para modificar las pasadas experiencias, utilizando la fantasía y la imaginación.

Además, se observa que la media aritmética del indicador relajación es de 0,396. Ubicándolo en la categoría bajo, lo cual indica que el personal directivo de los colegios nacionales de educación primaria del distrito Los Olivos de la provincia de Lima, Perú en tiempos de la pandemia del coronavirus COVID-19, posee un bajo conocimiento en cuanto a relajación. Esto representa un aspecto negativo para el desarrollo de PNL, ya que esta se soporta en la relajación, como una técnica idónea para disponer algunos programas, Gamboa et al. (2017) señalan que cuando se está en relajación, el sistema cerebral está en gran medida reparado, pues su energía esta armonizada y los potenciales eléctricos se encuentran más constantes. Cuando las personas se encuentran tranquilas y relajadas, son capaces de elegir que cosas son las que desean que su cerebro procese y también lo hace más rápido pues dispone de más energía y tiene menos desgaste.

Por otro lado, se observa que la media aritmética del indicador calibración es de 0,438, ubicándolo en la categoría mediano, esto indica que el personal directivo de los colegios nacionales de educación primaria del distrito Los Olivos de la provincia de Lima en tiempos de la pandemia del coronavirus COVID-19, posee un mediano conocimiento acerca de la estrategia calibración. Esto representa un inconveniente para la comunicación del personal directivo ya que, según Martínez y Ramos (2019) calibrar consiste en lograr hacer conscientemente algo que normalmente hacemos de manera inconsciente, perfeccionando nuestras habilidades en virtud de leer el cerebro en el otro sujeto con el que se pretende comunicarnos. La PNL plantea que cuando esta estrategia (calibración) se optimiza y desarrolla, se logra la excelencia y la maestría en la comunicación.

Igualmente se observa que, la media aritmética del indicador modelaje es de 0,375, ubicándose en la categoría bajo según el baremo. Esto indica que el personal directivo de los colegios nacionales de educación primaria del distrito Los Olivos de la provincia de Lima en tiempos de la pandemia del coronavirus COVID-19, posee bajo conocimiento acerca de modelaje, por su parte presenta un inconveniente para la comunicación del personal directivo ya que, según Gamboa et al. (2017), el modelaje es una técnica que fundamenta en estudiar los cuatro ejes fundacionales sobre lo que se cimenta el actuar del ser humano: la conducta visible, las habilidades, las características del lenguaje y los sistemas de creencias. Los autores previamente citados plantean que, en la PNL, el modelaje emplea los comportamientos de personas excelentes en un área específica, de forma tal que los aprendices puedan obtener aquellas estrategias que le han permitido esa excelencia y mejorar su propia ejecución.

También se considera que, la media aritmética del indicador sincronización es de 0,146, ubicándolo en la categoría muy bajo según el baremo. Esto indica que el personal directivo de los colegios nacionales de educación primaria del distrito Los Olivos de la provincia de Lima en 
tiempos de la pandemia del coronavirus COVID-19, posee un conocimiento muy bajo acerca de la sincronización presentando una apariencia negativa en referencia a la comunicación del personal directivo, puesto que la sincronización según Monterrosa et al. (2020) es una técnica empleada por la PNL para crear un clima de confianza entre los sujetos que se comunican, idóneo para obtener información y dirigir una conversación, en el que el interlocutor se percibe aceptado como es.

Además, es importante señalar que, estos resultados son similares a los de Sánchez (2020) quien evidenció en su estudio, como las estrategias de PNL logran proporcionar una comunicación efectiva entre los directores y sus compañeros de trabajo, optimando el entorno de labores en una organización para el logro eficaz de los objetivos de una institución. Por ello, si se realiza un avance permanente del desarrollo y ejecución de estas, las organizaciones educativas tendrían bastante éxitos en el alcance de los objetivos y la comunicación entre los directores y los empleados se perfeccionará, trayendo grandes beneficios a todos los involucrados directos del proceso educativo, directivos, docentes y estudiantes, lo cual es muy necesario en los actuales tiempos de pandemia.

Tabla 3. Distribución de medias aritméticas de los indicadores y de la dimensión estrategias de la Programación Neurolingüística.

\begin{tabular}{|c|c|c|c|c|c|c|c|}
\hline \multirow{2}{*}{$\begin{array}{c}\text { Variable } \\
\text { Dimensión }\end{array}$} & \multicolumn{7}{|c|}{ Programación Neurolingüística } \\
\hline & \multicolumn{7}{|c|}{ Estrategias de la PNL } \\
\hline Indicadores & Anclaje & Re encuadre & $\begin{array}{c}\text { Sub- } \\
\text { modalidades }\end{array}$ & Relajación & Calibración & Modelaje & $\begin{array}{l}\text { Sincroniza- } \\
\text { ción }\end{array}$ \\
\hline $\begin{array}{c}\bar{x} \\
\text { Indicador } \\
\end{array}$ & 0,396 & 0,375 & 0,458 & 0,396 & 0,438 & 0,375 & 0,146 \\
\hline $\begin{array}{l}x \\
\text { Dimensión }\end{array}$ & & & & 0,369 & & & \\
\hline
\end{tabular}

Fuente: Elaboración propia.

\section{Conclusiones}

Se analizaron los aspectos de la PNL que conoce el personal directivo de los colegios nacionales de educación primaria del distrito Los Olivos de la provincia de Lima, Perú, en tiempos de la pandemia del coronavirus COVID-19, concluyendo que éste personal tiene un mediano conocimiento acerca de la PNL, lo cual resulta un aspecto negativo en cuanto a la comunicación de dicho personal, ya que no conocen en su totalidad la PNL.

Por otro lado, se identificaron las bases de la Programación Neurolingüística conocidas por el personal directivo de los colegios nacionales de educación primaria del distrito Los Olivos de la provincia de Lima en tiempos de la pandemia del coronavirus COVID-19, concluyendo que dicho personal posee un bajo conocimiento de estas, lo cual representa una dificultad para una efectiva comunicación, ya que estas bases permiten descodificar los lenguajes procedentes de las distintas zonas del cerebro.

Así mismo, se caracterizaron las propuestas fundamentales de la PNL, conocidas por el personal directivo de los colegios nacionales de educación primaria del distrito Los Olivos de la 
La Programación Neurolingüística en los directivos de las organizaciones educativas peruanas en tiempos de pandemia.

provincia de Lima, concluyendo que éste personal posee un mediano conocimiento acerca de dichas propuestas, lo cual representa un aspecto negativo ya que estas propuestas son de vital importancia puesto que muchas veces se encuentran personas que presentan problemas para decir lo que pretenden comunicar a los demás. Pues si se aprenden a interpretar los modelos, se consiguen derrumbar los obstáculos de la comunicación.

Además, se estudiaron las estrategias de la PNL, reconocidas por el personal directivo de los colegios nacionales de educación primaria del distrito Los Olivos de la provincia de Lima en tiempos de pandemia, concluyendo que el personal directivo posee bajo conocimiento acerca de las estrategias de la PNL, lo cual representa un obstáculo para una comunicación efectiva, ya que desconocen las características de dichas estrategias, las cuales son obvias para una buena comunicación. La PNL establece que el éxito consiste simplemente en emplear la estrategia más idónea.

Finalmente, se propone, un entrenamiento con la PNL que posibilite el desarrollo de habilidades con la que se puedan concebir resultados que optimicen la comunicación del personal directivo de los colegios referidos en los actuales tiempos de pandemia.

\section{Referencias Bibliográficas}

Álvarez, Rina; Bernabé, Rosa; Gálvez, Mario. (2019). Estilos de aprendizaje basado en el modelo de programación neurolingüística y rendimiento académico de los estudiantes del quinto de secundaria. Ciencia y Desarrollo. 21 (2), Perú. (Pp. 4347). Disponible en: https://bit.ly/3cNBSxd

Arbeláez-Campillo, Diego; Villasmil, Jorge. (2020). Escenarios prospectivos de un nuevo orden internacional que se vislumbra luego de la pandemia covid-19. Telos: revista de Estudios Interdisciplinarios en Ciencias Sociales. 22 (3), Venezuela. (Pp. 494508). https://doi.org/10.36390/telos223.02

Arriagada, Cristina. (2018). Relación entre habilidad de metaconocimiento emocional, valoración ante la vida y nivel de ansiedad de funcionarios de un colegio de la ciudad de Concepción. Revista Reflexión e Investigación Educacional. 1 (2), Chile. (Pp. 3551). https://bit.ly/3mllQxz

Blanco, Sandra; Cerdas, Virginia; García, José. (2021). Clima organizacional en centros educativos públicos costarricenses: un análisis multifactorial. Revista Educación. 45 (1), Costa Rica. (Pp. 183-199). https://doi.org/10.15517/REVEDU.V45I1.41927

Burgos, Mildred. (2018). Praxis del liderazgo femenino y la programación neurolingüística (PNL) en el sector salud de la Guajira en Colombia. Consensus (Santiago)-Revista interdiscipliaria de investigación. 2 (3), Chile. (Pp. 21-46). Disponible en: https://bit.ly/3mic8f9

Carrión, Salvador. (2010). La escritura neurolingüística de las emociones. El desarrollo de la inteligencia emocional con PNL. Editorial: Excelencia Humana. España.

Cobo-Rendón, Rubia; Vega-Valenzuela, Andrés; García-Álvarez, Diego. (2020). Consideraciones institucionales sobre la Salud Mental en estudiantes universitarios durante la pandemia de Covid-19. CienciAmérica. 9(2), Ecuador. (Pp. 277-284). https://doi.org/10.33210/ca.v9i2.322 
Délano, Paul. (2020). Coronavirus y personal de la salud. Revista de otorrinolaringología y cirugía de cabeza y cuello. 80 (1), Chile. (Pp.7-17). http://dx.doi.org/10.4067/S0718-48162020000100007

Deminco, Marcus. (2019). Programación Neurolingüística. Comenzando por el principio. Clube de Autores Editores. Brasil.

Dilts, Robert; DeLozier, Judith. (2016). PNL II: Programación neurolingüística, la siguiente generación. El Grano de Mostaza Ediciones. España.

Douat, Gérard. (2017). PNL Programación neurolingüística. Parkstone International. Perú.

Estupiñan, Jesús; de Mora, Karina. (2017). La influencia de la programación neurolingüística en estudiantes universitarios en la República de Ecuador. LUZ. 16 (1), Cuba. (Pp. 104113). Disponible en: https://bit.ly/3sPxvr7

Flores, Zulma. (2018). Aplicación de PNL al modelo educativo del TECNM para el fomento y desarrollo de inteligencias múltiples en la mejora continua del proceso enseñanza aprendizaje de alumnos universitarios. Congreso Internacional de Ciencias Sociales. México. Disponible en: https://bit.ly/3sPg49X

Gamboa, María; García, Yenny; Ahumada, Vicky. (2017). La Programación Neurolingüística. Editorial: Libros Universidad Nacional Abierta y a Distancia. Colombia. Disponible en: https://bit.ly/3ulBiwB

Hernández, Roberto; Fernández, Carlos; Baptista, Pilar. (2018). Metodología de la Investigación. Mc. Graw Hill. México.

Huilca, Wilson. (2017). Actividades de programación neurolingüística como fuente de afrontamiento del estrés laboral, en personal docente de la Unidad Educativa Princeton. Alternativas. 18 (3), Guayaquil. (Pp. 41-48). Disponible en: https://bit.ly/3sRnf1r

López, Manuel. (2020). Ser estudiante LGBTI+ durante la pandemia COVID-19. Revista Latinoamericana de Estudios Educativos. 50 (Especial), México. (Pp. 225-230). Disponible en: https://bit.ly/3wnyTmR

López, Silvina; Vásquez, Claudio; Valdez, Liliana. (2020). Estudio socioeducativo de caso mejora del clima laboral en guarderías mediante la comunicación efectiva al utilizar técnicas de programación neurolingüística. RIDE Revista Iberoamericana para la Investigación y el Desarrollo Educativo. 11 (21), México. https://doi.org/10.23913/ride.v11i21.690

Maldonado-Vicente, Gisella. (2017). Influencia del coaching con programación neurolingüística en el desarrollo de competencias en los directivos. San Martín Emprendedor, 4 (2), Perú. (Pp. 68-79). Disponible en: https://bit.ly/2R8JWjX

Martínez, Eliana; Ramos, Yisela. (2019). Técnicas de programación neurolingüística (PNL) para el aprendizaje de histología en los estudiantes de segundo semestre de la Carrera de Laboratorio Clínico e Histopatológico. Tesis de Maestría. Maestría en Ciencias de la Educación Mención Biología. Universidad Nacional de Chimborazo, Ecuador. Disponible en: https://bit.ly/3fMC95g

Mayorga-Jácome, Lilia; Rosero-Morales, Elena (2020). Modelo de programación neurolingüística para el desarrollo del comportamiento social de los estudiantes de educación general básica superior. Dominio de las Ciencias. 6 (2), Ecuador. (Pp. 587-615). Disponible en: https://bit.ly/3uiejT6 
La Programación Neurolingüística en los directivos de las organizaciones educativas peruanas en tiempos de pandemia.

Monasterio, Dilia; Briceño, Magally. (2020). Educación mediada por las Tecnologías: Un desafío ante la coyuntura del Covid-19. Observador del Conocimiento. 5 (1), Venezuela.

(Pp. 136-148). Disponible en: https://bit.ly/3fDkJlv

Monterrosa, Nely; Ochoa, Eduin; Paéz, Yesevith. (2020). Programación neurolingüística en instituciones educativas: Una mirada desde los sistemas de representación sensorial. Consensus (Santiago)-Revista interdisciplinaria de investigación, 4 (3), Chile. (Pp. 03-33). Disponible en: https://bit.ly/2PArdx9

Montiel, Oscar; Flores, Anel; Ávila, Ernesto; Sierra, Susana. (2021). "tengo que sobrevivir": relato de vida de tres jóvenes micro emprendedores bajo COVID-19. Telos: revista de Estudios Interdisciplinarios en Ciencias Sociales. 23(1), Venezuela. (Pp.67-84). https://doi.org/10.36390/telos231.06

Morles, Lorena; Camargo, Esmerlis; Batista, Judeira; Muñoz, Miriam; Rodríguez, Jesús; MezaAndrade, Lina. (2017). Influencia del Liderazgo integrador y programación neurolingüística en gerentes del sector petrolero. Revista Espacios. 38 (58), Venezuela. Disponible en: https://bit.ly/3dxJVxl

Muñoz, Dairo. (2019). La Programación Neurolingüística como recurso didáctico en el aula virtual. Pensamiento udecino, 1 (1), Colombia. Disponible en: https://bit.ly/3ulhl9f

Navarrete, Alicia. (2017). El enfoque de pensamiento sistémico y la programación neurolingüística como factor clave de sustentabilidad en las organizaciones turísticas. Revista Iberoamericana de Producción Académica y Gestión Educativa. 4 (7), México. (Pp. 65-82). Disponible en: https://bit.ly/39FdUT4

Redford, Clara. (2017). PNL: Programación neurolingüística: Una guía práctica y sencilla para iniciarse en la programación neurolingüística. Editorial: Robinbook. España.

Rojas, Vivian; Calle, Ruth. (2019). Programación neurolingüística en la generación de redes afectivas para la resolución de conflictos en espacios pedagógicos. Universidad Ciencia y Tecnología. 2 (2), Venezuela. (Pp. 5-10). Disponible en: https://bit.ly/3wsgFk8

Romero, Mireya. (2020). Depression and the meaning of life in university students in times of pandemic. [Depresión y sentido de la vida en estudiantes universitarios en tiempos de pandemia]. International Journal of Educational Psychology. 9 (3), España. (Pp. 223-242). https://doi.org/10.17583/ijep.2020.6784

Romero, Rosario; Barboza, Luis; Sánchez, Miguel. (2015). La Gerencia estratégica fundamentada con la programación neurolingüística. Omnia. 21 (1), Venezuela. (Pp. 115-128). Disponible en: https://bit.ly/3cL7inZ

Rosario-Rodríguez, Adam; González-Rivera, Juan; Cruz-Santos, Andrés; Rodríguez-Ríos, Luismiguel. (2020). Demandas Tecnológicas, Académicas y Psicológicas en Estudiantes Universitarios durante la Pandemia por COVID-19. Revista Caribeña de Psicología. 4 (2), Puerto Rico. (Pp. 176-185). https://doi.org/10.37226/rcp.v4i2.4915

Sánchez, Lida. (2020). Liderazgo corporativo hacia una comunicación asertiva con herramientas de PNL. Tesis de Maestría. Facultad de Comunicación y Lenguaje. Pontificia Universidad Javeriana. Colombia. Disponible en: https://bit.ly/3dvJSC5 
Sanz, Ismael; Sáinz, Jorge; Capilla, Ana. (2020). Efectos de la crisis del coronavirus en la educación. Organización de Estados Iberoamericanos para la Educación, la Ciencia y la Cultura (OEI). España. Disponible en: https://bit.ly/3fFJ3cM

UNESCO. (2020). 290 million students out of school due to COVID-19: UNESCO releases first global numbers and mobi-lizes response. Disponible en: https://bit.ly/2PVc4WO

Vanga, María; Fernández, Adalberto. (2016). Programación neurolingüística para fomentar la dirección afectiva, creatividad y comunicación en el aula. Revista AUC, (37), Guayaquil. (Pp. 43-50). Disponible en: https://bit.ly/3dAYOKe

Velavan, Thirumalaisamy; Meyer, Christian. (2020). La epidemia de COVID-19. Tropical Medicine \& International Health. 25 (3), Reino Unido. (Pp. 278-280). https://doi.org/10.1111/tmi.13383

Vivas, Ramiro; Cabanilla, Enrique; Vivas, Walter. (2019). Relación entre los estilos de aprendizaje y el rendimiento académico del estudiantado de la carrera de Ingeniería Agronómica de la Universidad Central del Ecuador. Revista Educación. 43 (1), Costa Rica. (Pp. 468-482). https://doi.org/10.15517/REVEDU.V43I1.28439 